ARTICLE

\title{
Stereoselective hydrogen atom transfer to acyclic radicals: a switch enabling diastereodivergent borylative radical cascades
}

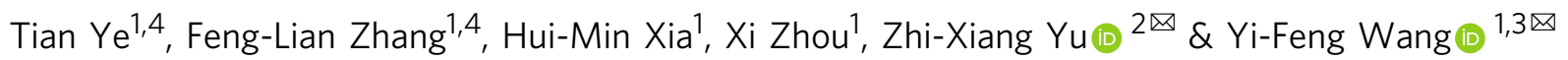

Radical cascade reactions are powerful tools to construct structurally complex molecules. However, the stereochemical control of acyclic radical intermediates remains a persistent challenge, due to the low differentiation between the two faces of these species. This hurdle further makes stereodivergent synthesis rather more difficult to be accomplished, in particular for intermediates resulted from complex cascades. Here we report an efficient strategy for stereoselective hydrogen atom transfer (HAT) to acyclic carbon radicals, which are generated via $\mathrm{N}$-heterocyclic carbene (NHC)-boryl radicals triggered addition-translocationcyclization cascades. A synergistic control by the $\mathrm{NHC}$ subunit and a thiol catalyst has proved effective for one facial HAT, while a $\mathrm{Znl}_{2}$-chelation protocol allows for the preferential reaction to the opposite face. Such a stereoselectivity switch enables diastereodivergent construction of heterocycles tethering a boron-substituted stereocenter. Mechanistic studies suggest two complementary ways to tune HAT diastereoselectivity. The stereospecific conversions of the resulting boron-handled products to diverse functionalized molecules are demonstrated.

\footnotetext{
${ }^{1}$ Department of Chemistry, University of Science and Technology of China, 96 Jinzhai Road, 230026 Hefei, Anhui, China. ${ }^{2}$ Beijing National Laboratory for Molecular Sciences (BNLMS), Key Laboratory of Bioorganic Chemistry and Molecular Engineering of Ministry of Education, College of Chemistry, Peking University, 100871 Beijing, China. ${ }^{3}$ State Key Laboratory of Elemento-Organic Chemistry, Nankai University, 300071 Tianjin, China. ${ }^{4}$ These authors

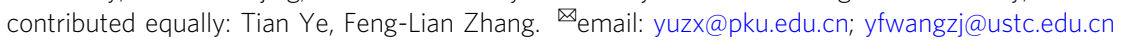


C onstruction of complex organic molecules in step- and atom-economic manners with high levels of stereoselectivity control is a preeminent goal in synthetic chemistry $^{1,2}$. Radical cascade reactions represent one of the most efficient strategies to assemble densely functionalized architectures from simple starting materials in one step ${ }^{3-5}$. However, the stereochemical control in these reactions remains an inherently difficult issue when acyclic carbon stereocenters are created through complex cascade processes, due to the persistent challenge associated with the low differentiation between the two faces of acyclic alkyl radicals ${ }^{6,7}$. This hurdle also makes the stereodivergent synthesis, which is highly demanded in medicinal studies $^{8}$, rather difficult to be achieved.

Hydrogen atom transfer (HAT) reactions often serve as a key step to terminate a radical cascade sequence through the reduction of the resulting radical intermediate ${ }^{9-11}$. However, HAT to acyclic radical intermediates with high levels of diastereoselectivity is a subject of persistent challenge ${ }^{12-15}$. In this context, some stereoselective HAT approaches have been reported, which include, for example, introducing different steric or electronic interactions in acyclic substrates (Fig. 1a). In addition, chelation by the use of Lewis acids has been applied so that the corresponding acyclic systems become rigid and high diastereoselectivity is consequently possible to be realized ${ }^{16,17}$. Although these approaches have proven effective in certain cases, some limitations and drawbacks still remain: (i) most methods are restricted to specific substrates with the preinstallation of different functional groups onto specific positions for steric, electronic, or complexation purposes, but such tactics are hardly applied in the reduction of radicals derived from complex radical cascades; (ii) stereocontrol is highly temperature-dependent and relatively lower temperature (usually below room temperature) is often necessary to ensure high diastereoselectivity, but this becomes disfavored for radical cascades that need high temperature ${ }^{18,19}$; (iii) diastereodivergent HAT reactions have only been reported in limited cases $^{20}$, and a general strategy for diastereodivergency is still lacking. Therefore, developing new strategies that can render

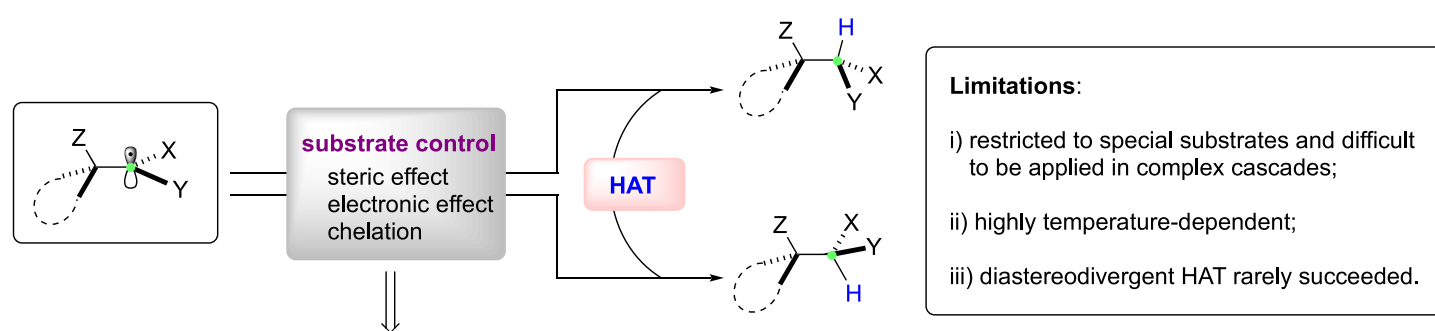

A challenging goal:

development of strategically new

and stereodivergent modes

b

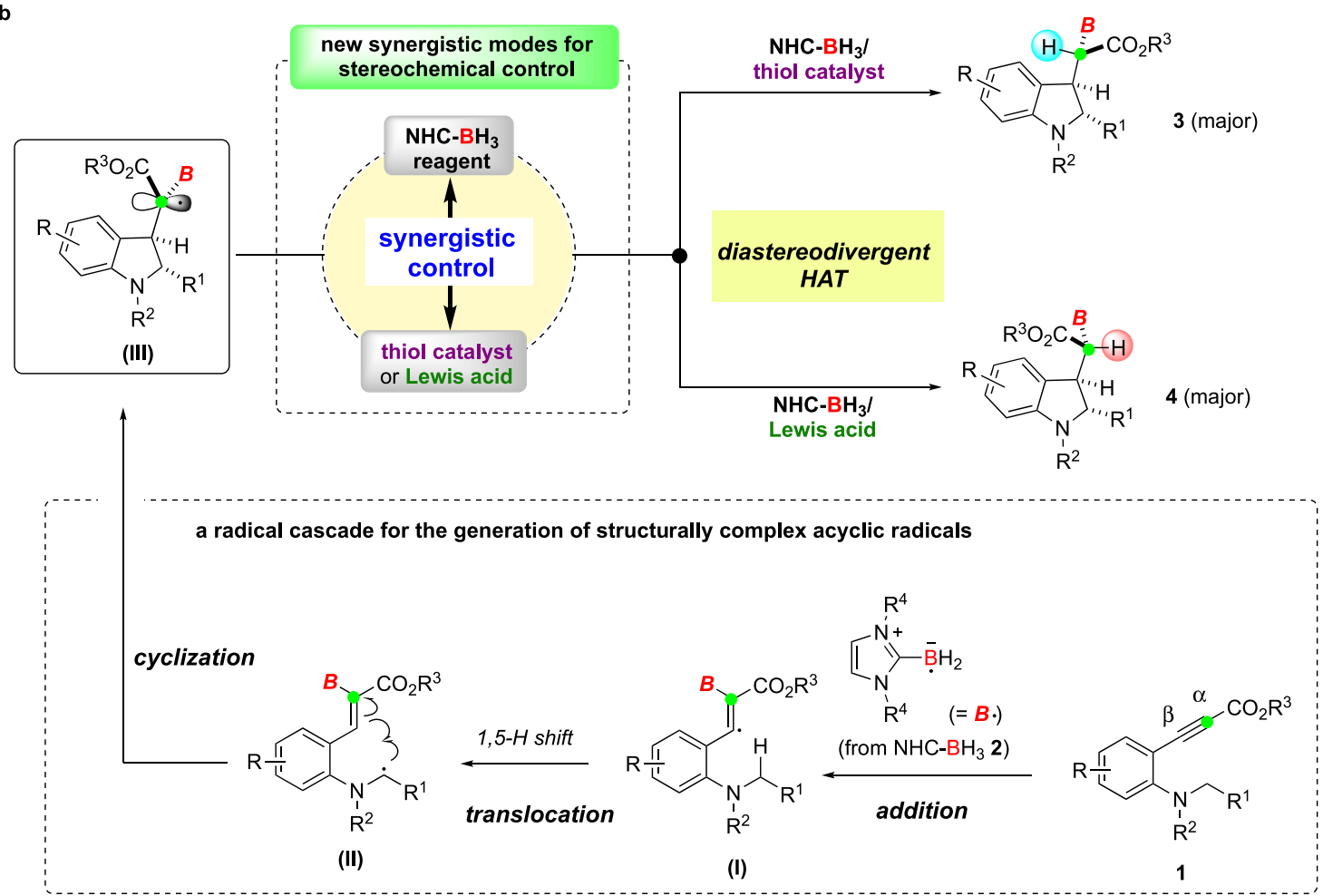

Fig. 1 Diastereoselective HAT to acyclic radicals and the applications in synthesis. a Reported diastereoselective HAT of acyclic radicals methods mainly

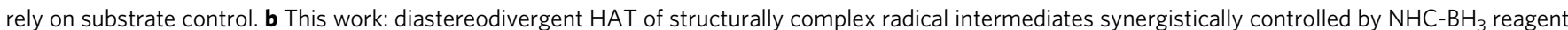
with either a thiol catalyst or a Lewis acid. NHC N-heterocyclic carbene, HAT hydrogen atom transfer. 
the reduction of acyclic radical intermediates to be stereoselectively distinguishable and switchable is highly desirable for advancing radical chemistry in synthesis.

$\mathrm{N}$-heterocyclic carbene (NHC)-borane complexes have recently been developed as an important class of hydrogen atom donor, as pioneered by Fensterbank, Lacôte, Malacria, and Curran ${ }^{21}$. These complexes feature easy accessibility, low toxicity, and broad applicability. Moreover, the group of Curran and Taniguchi ${ }^{22-25}$, our group $26-29$, and others ${ }^{30-33}$ disclosed that these complexes are viable for the synthesis of organoboron molecules via radical cascade reactions, wherein both the boryl moiety and hydrogen atom are transferred to products. A polarity reversal catalyst ${ }^{34,35}$, such as thiol compound, is sometimes required to maintain efficient chain propagation. It is known that NHC molecules with various substitutions and stereochemistry are either commercially available or can be synthesized readily. Therefore, the NHC subunit would be considered as an important and tunable element for stereocontrol, where various potential factors, including steric or electronic effect, weak interactions between catalysts or substrates, and complexation with Lewis acid, would be used to modulate HAT stereoselectivity. Meanwhile, the NHC-boryl moiety is installed during the reaction process, thereby avoiding the prior installation of specific stereoselectivity-determining functionalities into substrates, which is desired in complex radical cascade reactions.

With this in mind, we hypothesized an NHC-boryl radical triggered radical addition-translocation-cyclization (RATC) cascade $^{36-39}$ that could generate a boron-substituted structurally complex acyclic radical intermediate. As shown in Fig. 1b, the reaction starts with the a-addition of an NHC-boryl radical to arylpropiolates $\mathbf{1}^{28}$, followed by 1,5-hydrogen atom shift and intramolecular 5-exo cyclization, yielding an indoline ring with trans-stereochemistry of cyclic substituents. The following HAT to the resulting acyclic radical III with high stereocontrol is perceived to be a major challenge for achieving the goal of stereoselective and stereodivergent synthesis of boron-tethered indolines.

Here we report our realization of diastereoselective HAT to this acyclic radical, which is accomplished by a synergistic control of $\mathrm{NHC}-\mathrm{BH}_{3}$ reagents together with either a thiol catalyst or a Lewis acid. Notably, these two modes operate in complementary ways that offer a stereochemistry switch to enable diastereodivergent synthesis of borylated products $\mathbf{3}$ and $\mathbf{4}$. Mechanistic investigations of the detailed mechanisms are carried out to understand how the present HAT reactions take place and the origins of stereochemistry. Significantly, the formed boron-tethered indoline derivatives would be of great interest in synthetic and medicinal chemistry, given the fact that indoline is a core motif in numerous biologically relevant molecules ${ }^{40,41}$. Moreover, the boron moiety can be used in stereospecific diversifications ${ }^{42-44}$, providing direct and economical accesses to various functionalities through late-stage functionalizations, thus circumventing the traditional lengthy de novo synthesis.

\section{Results}

$\mathrm{NHC}-\mathrm{BH}_{3} /$ thiol catalyst-controlled stereoselective HAT to acyclic radicals generated via borylative RATC. We commenced our study by investigating the reaction of $\mathbf{1 a}$ and $\mathrm{NHC}-\mathrm{BH}_{3} \mathbf{2 a}$ in the presence of di-tert-butyl hyponitrite (TBHN) as the radical initiator. The reaction took place as expected, affording diastereomers 3a-Me and 4a-Me in 52\% combined yield and a 67:33 diastereomeric ratio (dr) (Table 1, entry 1). Guindon has found that the exocyclic effect plays a vital role in the stereoselective reduction of carbon-centered radicals locating exo to a heterocycle ${ }^{14}$. However, the low dr value obtained in our reaction suggested that such an effect was not significant for the reduction of radical III.

Next, we sought to examine various $\mathrm{NHC}-\mathrm{BH}_{3}$ complexes and thiol catalysts to improve the diastereoselectivity. The use of $\mathbf{2 b}$ instead of 2a led to a lower conversion, but with a slightly increased dr (entry 2). Interestingly, when a thiol compound (thiol $\mathbf{A}, \mathbf{B}$, or $\mathbf{C}$ ) was employed as a polarity reversal catalyst $\mathrm{s}^{34,35}$, both the product yield (52-79\%) and stereoselectivity (dr, 90:1092:8) were enhanced (entries 3-5). Furthermore, using orthosubstituted thiophenols increased the dr (93:7 to 94:6) while maintaining high product yields (entries 6-8). However, 2,4,6triisopropyl substituted thiol $\mathbf{G}$ resulted in a much lower $\mathrm{dr}$ (88:12) although good product yield could be retained (entry 9). Eventually, thiol $\mathbf{H}$ was found to be optimal, furnishing $\mathbf{3 a}-\mathbf{i}-\mathbf{P r}$ in excellent yield and $\mathrm{dr}$ (entry 10). Using thiol $\mathbf{H}$ as the catalyst, the reaction with 2a resulted in a decrease of dr to 88:12 (entry 11), implying that the isopropyl group of $\mathrm{NHC}-\mathrm{BH}_{3} \mathbf{2} \mathbf{b}$ is also crucial to maintain high diastereoselectivity. The reaction of $2 \mathrm{c}$ bearing a 2,6-diisopropylphenyl motif led to nearly no reaction (entry 12), presumably due to the increased difficulty in the addition step. These findings support a fact that the stereoselectivity of HAT to acyclic radical III is cooperatively controlled by the substituents of both $\mathrm{NHC}-\mathrm{BH}_{3}$ and the thiol catalyst. When the radical initiator was changed to 2,2-azobisisoheptonitrile (ABVN), 3a-iPr was formed in 93\% yield and 95:5 dr (entry 13). However, the employment of azobisisobutyronitrile (AIBN) and azobiscyclohexanecarbonitrile $(\mathrm{ACCN})$, which require elevated temperature $\left(80-95^{\circ} \mathrm{C}\right)$ for initiation, caused decreased diastereoselectivity (entries 14-15). The control experiment showed that no reaction occurred in the absence of a radical initiator (entry 16), verifying a radical reaction mechanism of this transformation.

The generality of this $\mathrm{NHC}-\mathrm{BH}_{3} /$ thiol catalyst-controlled diastereoselective synthesis of product 3 was examined (Fig. 2). In most cases, high levels of diastereoselectivity ( $\mathrm{dr}>92: 8$ ) could be obtained. An array of functional groups on the 2-aryl ring (for $\mathbf{3 c}-\mathbf{3 e}$ ) and indoline framework (for $\mathbf{3 h}$ and $\mathbf{3 i}$ ) were incorporated. Both naphthyl (for $\mathbf{3}$ ) and thiophenyl (for $\mathbf{3 g}$ ) motifs were compatible. The reaction of $\mathbf{1} \mathbf{j}$ delivered a pyrrolo[2,3-b]pyridine framework (for $\mathbf{3 j}$ ), albeit with a slightly decreased diastereoselectivity. A series of alkyl groups could be used as the $\mathrm{R}^{2}$ substituent (for $\mathbf{1 k}-\mathbf{1 m}$ ) on the nitrogen atom, furnishing desired products in both excellent yields and diastereoselectivity. When the $\mathrm{R}^{2}$ substituent was replaced with a phenyl ring, the reaction could produce the expected product $3 \mathbf{n}$ in $82 \%$ yield, but with a moderate stereoselectivity $(86: 14 \mathrm{dr})$. However, no reaction occurred for the hydrogen substituted one (for 1o). The present protocol allowed for the construction of a tetracyclic framework (for 3p) with no detrimental effect on diastereoselectivity. The borylative cascade reactions of $\mathbf{1 q}$ and $\mathbf{1 r}$, however, became less efficient ( $<25 \%$ yield of cyclized products) and hydroboration of the alkyne moiety ${ }^{23,45,46}$ was observed as a competing reaction pathway. This is presumably due to the disfavored 1,5-hydrogen atom transfer of the corresponding alkenyl radical $\mathbf{I}$ that makes the reduction by the thiol catalyst possible. To our delight, the desired cascade reactions could be promoted by increasing the reaction temperature to $95^{\circ} \mathrm{C}$ with the use of ACCN as the radical initiator and using $\mathbf{2 a}$ as the boryl radical precursor, furnishing products $\mathbf{3 q}$ and $\mathbf{3 r}$ in $83 \%$ yield $(86: 14 \mathrm{dr})$ and $57 \%$ yield $(67: 33$ dr), respectively. In these transformations, 2a itself also acted as the hydrogen atom donor to maintain the radical chain process ${ }^{47}$. It was also found that the addition of a thiol catalyst was not effective at improving the $\mathrm{dr}$ value in this case (see Table S4 in the Supplementary Information). Likewise, the standard reaction protocol of $1 \mathrm{~s}$ led to no reaction, while the modified procedure provided the borylated product in $65 \%$ yield, albeit with low diastereoselectivity. 
Table 1 Optimization of the reaction conditions ${ }^{a}$.

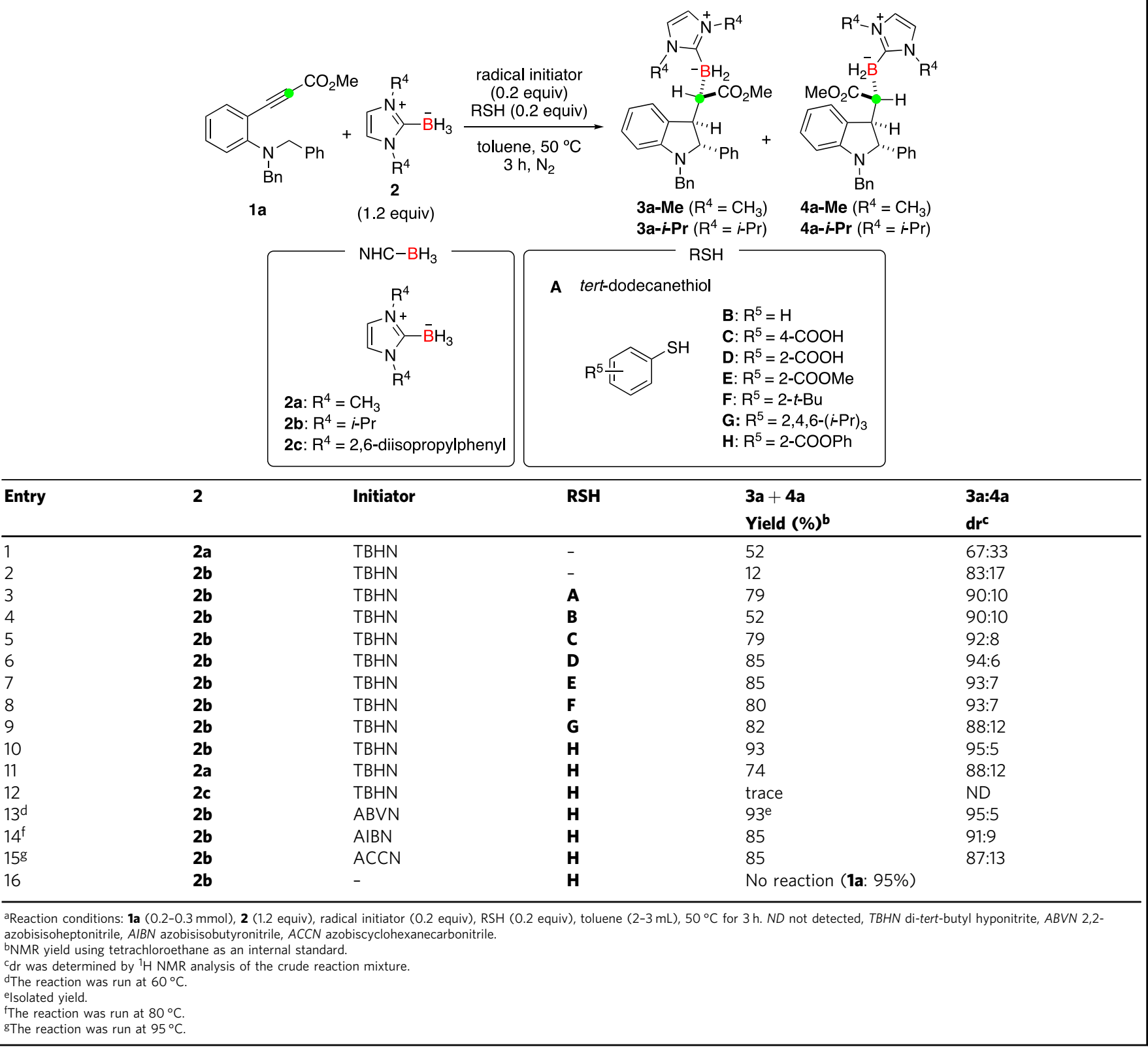

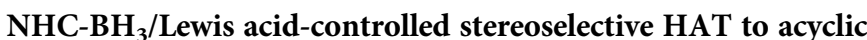
radicals generated via borylative RATC. Next, we questioned whether it is possible to reverse the stereoselectivity of the HAT process by changing the reaction conditions, leading to the preferential formation of diastereomers 4 . Considering that acyclic radical III contains some coordinative functionalities, such as methoxycarbonyl group, NHC subunit, and amino group, a range of Lewis acids have been examined. Eventually, it was found that the addition of $\mathrm{ZnI}_{2}$ as a chelating reagent and the employment of $\mathbf{2 d}$ as the boryl radical precursor could afford 4aa in good yield and excellent diastereoselectivity (3aa:4aa $=6: 94$, Table 2, entry $1)$. No thiol catalyst was required in this reaction, implying that acyclic radical III should abstract a hydrogen atom from $\mathbf{2 d}$ to propagate the radical chain process ${ }^{47}$. In this case, the formed radical intermediate III likely became more electrophilic by coordination of $\mathrm{ZnI}_{2}$ to the carbonyl group, thus promoting the
HAT process by more polarity matching. Reducing the amount of $\mathrm{ZnI}_{2}$ resulted in a dramatic decrease of $\mathrm{dr}$ (entries 2 and 3), and the reaction without $\mathrm{ZnI}_{2}$ even gave 3aa as the major product (entry 4). Other Lewis acids such as $\mathrm{MgCl}_{2}, \mathrm{MgI}_{2}, \mathrm{CuI}, \mathrm{ZnCl}_{2}$, $\mathrm{Me}_{3} \mathrm{Al}$, and $\mathrm{MgBr}_{2} \cdot \mathrm{OEt}_{2}{ }^{48}$ were found to be ineffective (entries 510). When $\mathbf{2 a}$ was used as the radical precursors instead of $\mathbf{2} \mathbf{d}$, the $\mathrm{dr}$ value was retained, although the yield was decreased to $37 \%$ (entry 11). The reaction of $\mathbf{2} \mathbf{b}$ led to only a trace amount of product (entry 12). These results indicate that the $\mathrm{ZnI}_{2}$ coordination with the triazole moiety of $\mathbf{2 d}$ may be beneficial to maintain the radical chain propagation, but the contribution in controlling the diastereoselectivity is not significant.

This $\mathrm{NHC}-\mathrm{BH}_{3} / \mathrm{ZnI}_{2}$ chelation-controlled diastereoselective synthesis showed broad substrate scope and functional group tolerance (Fig. 3). In most reactions, both moderate to good yields and high levels of $\mathrm{dr}(8: 92 \sim 6: 94)$ could be obtained (4aa, 4ha, 4ia, 


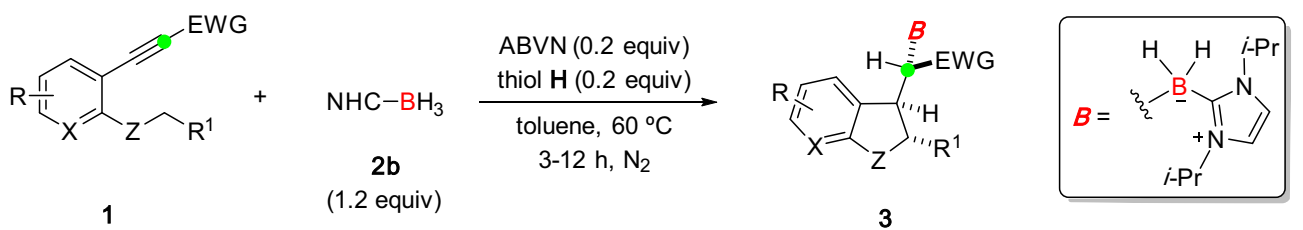

(1.2 equiv)

3

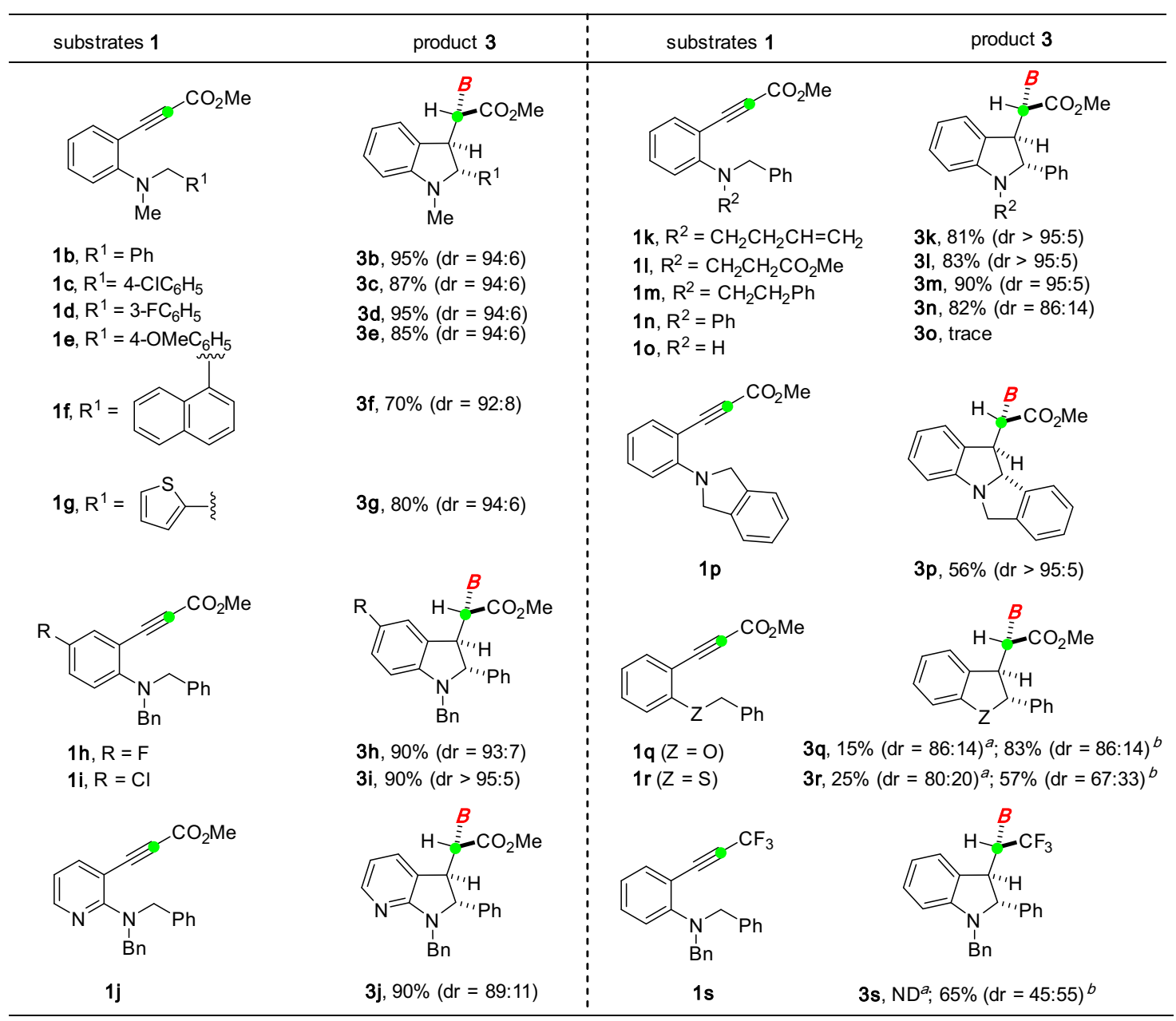

Fig. 2 NHC-BH 3 /thiol catalyst-controlled stereoselective synthesis of borylated molecules derived from RATC. Reactions were performed with substrate $\mathbf{1}$ ( $0.2-0.5 \mathrm{mmol}), \mathbf{2 b}$ (1.2 equiv), ABVN (0.2 equiv), and thiol $\mathbf{H}(0.2$ equiv) in toluene ( $2-3 \mathrm{~mL})$ at $60^{\circ} \mathrm{C}$ for $3-12 \mathrm{~h}$. The yield is recorded for the combination of diastereomers $\mathbf{3}$ and $\mathbf{4}$. dr refers to the ratio of 3:4. ND, not detected. aResults obtained with the reaction conducted under standard reaction conditions and a competing hydroboration reaction of the alkyne moiety was observed. besults obtained with the reaction conducted using $\mathbf{2} \mathbf{a}$ (1.2 equiv) and $\mathrm{ACCN}\left(0.5\right.$ equiv) at $95^{\circ} \mathrm{C}$.

4ta-4xa). However, when the $\mathrm{R}^{2}$ substituent was changed to other alkyl groups (for $\mathbf{1 b}$ and $\mathbf{1 1}$ ) or phenyl group (1n), the products were formed with low stereochemical outcomes. This implied that the benzyl group as $\mathrm{R}^{2}$ is crucial. In addition, replacing the terminal methoxycarbonyl group with a non-coordinating $\mathrm{CF}_{3}$ moiety resulted in a precipitous drop in diastereoselectivity (3sa:4sa $=$ 45:55), hinting the participation of the carbonyl moiety in the diastereo-determining step. Moreover, the presence of a strong coordinative pyridine ring (for $\mathbf{1 j}$ ) also interrupted the chelation, resulting in almost no diastereoselectivity.

Synthetic applications. The synthetic utility of this diastereodivergent RATC process was demonstrated (Fig. 4). Diastereomeric a-boryl carbonyls $3 \mathbf{i}$ and $4 \mathbf{i a}$ were converted to a series of useful building blocks based on the versatile chemistry of both the boryl and carbonyl moieties. For example, reduction of the ester group of $\mathbf{3 i}$ and $4 \mathbf{i a}$ followed by oxidation afforded diastereomeric 1,2-diols $\mathbf{5 a}$ and $\mathbf{5} \mathbf{a a}$ in good yields. Instead of oxidation, the treatment with $\mathrm{N}$-chlorosuccinimide (NCS) in the presence of pinacol and further protection of the hydroxy group provided useful pinacol boronic esters $\mathbf{6} \mathbf{a}$ and $\mathbf{6} \mathbf{a a}$, respectively. The subsequent coupling reaction with furan using Aggarwal's arylation protocol $^{49}$ delivered the corresponding $7 \mathbf{a}$ and $7 \mathbf{a a}$ in good efficiency. Moreover, homologation of $\mathbf{6 a}$ and $\mathbf{6 a}$ a also proceeded, furnishing alkyl boronic esters 8a and 8aa in good yields. It is worth mentioning that the starting materials $3 \mathbf{i}$ and $4 \mathbf{i a}$ were both used as a single diastereomer and the stereochemistry was retained in all these transformations, thus the resulting products were obtained with exclusive diastereoselectivity.

\section{Discussion}

Computational studies of diastereoselective HAT controlled by NHC- $\mathrm{BH}_{3} /$ thiol catalyst. To elucidate the diastereoselectivity of this HAT process, density functional theory (DFT) calculations 
Table 2 Examination of reaction parameters for the preferential formation of diastereomer $4 a^{a}$.<smiles>CC(=O)C#Cc1ccccc1N(Cc1ccccc1)Cc1ccccc1</smiles>

$1 a$<smiles>[BH3-]c1n(C)cn[n+]1C</smiles>

2d

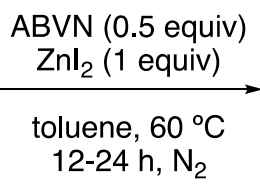

12-24 h, N

(1.2 equiv)<smiles>COC(=O)C1c2ccccc2N(Cc2ccccc2)C1c1ccccc1</smiles>

$3 a a$

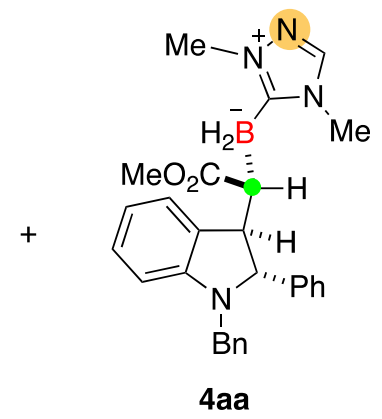

3aa:4aa

drc

$6: 94$

$12: 88$

$50: 50$

$75: 25$

$75: 25$

$67: 33$

$75: 25$

$75: 25$

$75: 25$

$56: 44$

$7: 93$

ND

Reaction conditions: $1(0.2-0.5 \mathrm{mmol}), \mathbf{2}$ (1.2 equiv), ABVN (0.5 equiv), Lewis acid ( $0-1$ equiv), toluene $(2-3 \mathrm{~mL}), 60^{\circ} \mathrm{C}$ for $12-24 \mathrm{~h}$. The yield is recorded for the combination of diastereomers $\mathbf{3}$ and $\mathbf{4}$ $\mathrm{dr}$ refers to the ratio of $\mathbf{3 : 4}$. ND, not detected.

$\mathrm{dr}$ refers to the ratio of $\mathbf{3 : 4}$. ND, not detected.
bNMR yield using tetrachloroethane as an internal standard.

c dr was determined by ${ }^{1} \mathrm{H}$ NMR analysis of the crude reaction mixture.

disolated yield.

3aa-Me and 4aa-Me were obtained.

were carried out at the (U)B3LYP(D3)/6-311+G(d,p)/SMD(toluene)//(U)B3LYP(D3)/6-31G(d,p) level of theory (Fig. 5). Firstly, the rotational barrier of $\mathrm{C}_{1}-\mathrm{C}_{2}$ bond in Int-III-A was roughly estimated by single-point energy calculations of the optimized structures with the frozen dihedral angles of $\mathrm{B}-\mathrm{C}_{1}-\mathrm{C}_{2}-\mathrm{C}_{3}$ (Supplementary Fig. 4). The results reveal that $\sim 7 \mathrm{kcal} / \mathrm{mol}$ of energy barrier is required for the free rotation of $\mathrm{C}_{1}-\mathrm{C}_{2}$ bond in Int-IIIA. This energy is lower than the activation Gibbs energies of HATs, which are 8.3 and $10.6 \mathrm{kcal} / \mathrm{mol}$, respectively. Therefore, the stereochemistry is determined by the relative HAT transition states, based on the Curtin-Hammett principle ${ }^{50}$. Then hydrogen atom transfer to Int-III-A was computed by using thiol $\mathbf{E}$ for simplification. The transition states leading to products $\mathbf{3 a}-\mathbf{i}-\mathbf{P r}$ and $\mathbf{4 a - i - P r}$ were located as TS-A-major and TS-A-minor, respectively (Fig. 5). Before reaching these two transition states, two complexes between Int-III-A and thiol $\mathbf{E}$ are formed. TS-Amajor is $2.3 \mathrm{kcal} / \mathrm{mol}$ more favorable than TS-A-minor in terms of Gibbs free energy, suggesting that $\mathbf{3 a - i}-\mathbf{P r}$ is the major product. This is consistent with the experimental observation (Table 1, entry 7). To find the origin of the stereochemistry preference for TS-A-major over TS-A-minor, distortion/interaction analysis ${ }^{51-53}$ (DIA) was performed by separating the transition states into two fragments, Int-III-A and thiol E. The distortion energy for TS-A-major is slightly higher than that of TS-Aminor (18.8 vs. $18.2 \mathrm{kcal} / \mathrm{mol})$, and a stronger interaction energy is observed in TS-A-major $\left(\Delta \Delta E_{\text {int }}=-2.0 \mathrm{kcal} / \mathrm{mol}\right)$. These imply the presence of different interaction energies that determines the diastereoselectivity. The interactions between Int-III-A and thiol $\mathbf{E}$ in TS-A-major and TS-A-minor were further analyzed by noncovalent interaction ${ }^{54,55}$ (NCI) and AIM analysis.
The results indicate that the lower interaction energy in TS-A-major is likely attributed to the $\mathrm{CH} / \pi$ attraction ${ }^{56,57}$ between $\mathrm{H}_{\mathrm{a}}$ on thiol $\mathbf{E}$ and the 2-phenyl moiety, which is absent in the competing TS-A-minor. This is further supported by the experimental finding that thiol $\mathbf{G}$ lacking such ortho-Cs $p^{2}-\mathrm{H}$ gave inferior diastereoselectivity (Table 1, entry 9).

Experimental and Computational Studies of Diastereoselective HAT Controlled by $\mathrm{NHC}_{-} \mathrm{BH}_{3} /$ Lewis Acid. Experimental and computational studies were then conducted to study how $\mathrm{ZnI}_{2}$ chelation-controlled HAT process takes place. Because Int-III-B is assumed to have a chelation with $\mathrm{ZnI}_{2}$ similar to that of product 4aa, we used ${ }^{1} \mathrm{H}$ NMR spectroscopy measurements to probe the possible chelation sites employing 4 aa as a model (Fig. 6). Upon addition of $\mathrm{ZnI}_{2}$ (0-1.5 equiv), the protons locating on the triazole moiety $\left(\mathrm{H}_{\mathrm{a}}, \mathrm{H}_{\mathrm{b}}\right.$ and $\left.\mathrm{H}_{\mathrm{c}}\right)$ are shifted downfield. In addition, the proton of methoxycarbonyl group $\left(\mathrm{H}_{\mathrm{d}}\right)$ and a-carbonyl proton $\left(\mathrm{H}_{\mathrm{e}}\right)$ also exhibit a downfield shift. These imply that both the triazole and carbonyl groups are involved in binding with $\mathrm{Zn}^{\mathrm{II}}$. It should be noted that protons at $\alpha$-positions of the nitrogen atom $\left(\mathrm{H}_{\mathrm{g}}, \mathrm{H}_{\mathrm{i}}\right.$, and $\left.\mathrm{H}_{\mathrm{i}}^{\prime}\right)$ have negligible changes, which indicates that the coordination of this aryl-substituted nitrogen atom to the zinc center is weak. Importantly, only a single set of ${ }^{1} \mathrm{H}$ NMR spectrum is observed in all cases, suggesting a fast dynamics of complexation/decomplexation between the free $\mathbf{4 a a}$ and $\mathrm{Zn}^{\mathrm{II}}-4 \mathrm{aa}$ chelates. A similar behavior of dynamics between free $2 \mathbf{d}$ and $\mathrm{Zn}^{\mathrm{II}}-\mathbf{2 d}$ was also observed (Supplementary Fig. 3). As such, the following DFT calculations employed Int-III bound to one molecular of $\mathrm{ZnI}_{2}$ and NHC-BH 3 2d ligated with another one 

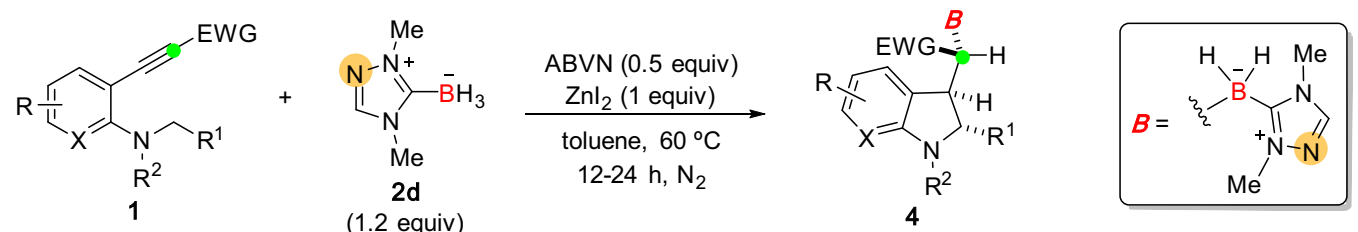

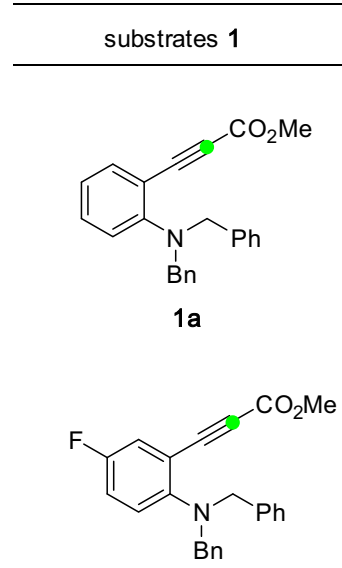

$1 \mathrm{~h}$<smiles>CC(=O)C#Cc1cc(Cl)ccc1N(Cc1ccccc1)Cc1ccccc1</smiles>

$1 \mathrm{i}$

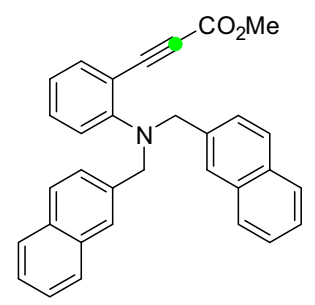

$1 t$

(1.2 equiv)

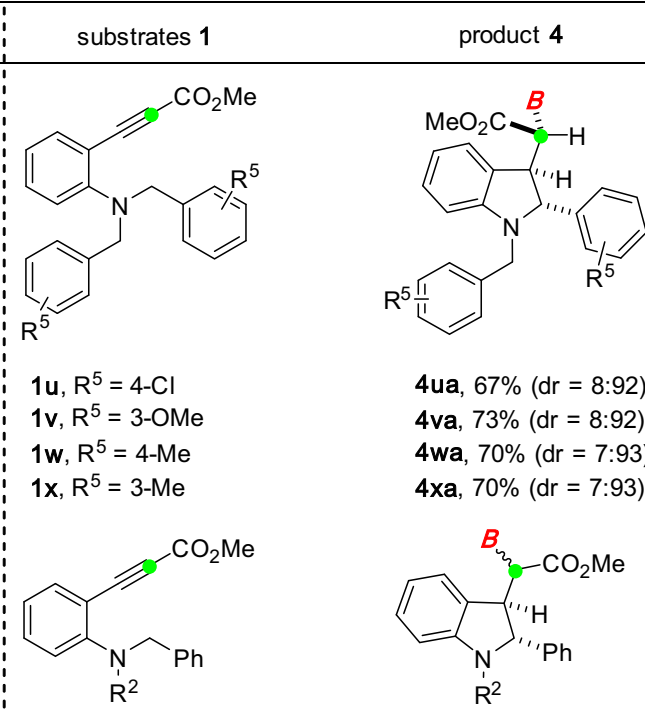

$1 \mathrm{~b}, \mathrm{R}^{2}=\mathrm{Me}$

1I, $\mathrm{R}^{2}=\mathrm{CH}_{2} \mathrm{CH}_{2} \mathrm{CO}_{2} \mathrm{Me}$

$1 \mathrm{n}, \mathrm{R}^{2}=\mathrm{Ph}$

4ia, $71 \%(d r=7: 93)$

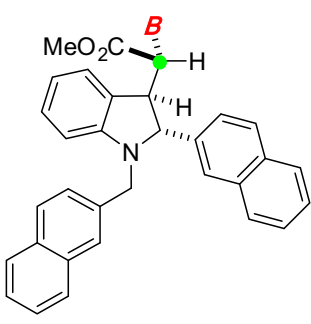

$4 \mathrm{ta}, 66 \%(\mathrm{dr}=6: 94)$
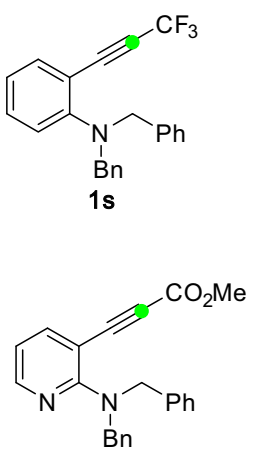

$1 \mathrm{j}$ 3ba+4ba, 44\% (dr = 67:33)

$3 l a+4 l a, 44 \%(d r=23: 77)$

3na+4na, $23 \%(\mathrm{dr}=72: 28)^{a}$<smiles>[B]OC(c1ccccc1)[C@H]1c2ccccc2N(Cc2ccccc2)[C@H]1c1ccccc1</smiles>

3sa+4sa, 64\% (dr $=45: 55)$<smiles>[B][C@@H](C(C)=O)[C@H]1c2cccnc2N(Cc2ccccc2)[C@H]1c1ccccc1</smiles>

$3 \mathrm{ja}+4 \mathrm{ja}, 47 \%(\mathrm{dr}=62: 38)$

Fig. $3 \mathbf{~ N H C - B H} / \mathbf{Z n l}_{2}$-controlled stereoselective synthesis of borylated molecules derived from RATC. Reactions were performed with substrate $\mathbf{1}$ ( $0.2-0.5 \mathrm{mmol}), \mathbf{2 d}$ (1.2 equiv), ABVN ( 0.5 equiv), and $\mathrm{ZnI}_{2}$ (1 equiv) in toluene $\left(2-3 \mathrm{~mL}\right.$ ) at $60^{\circ} \mathrm{C}$ for $12-24 \mathrm{~h}$. The yield is recorded for the combination of diastereomers $\mathbf{3}$ and $\mathbf{4}$. $\mathrm{dr}$ refers to the ratio of $\mathbf{3}: \mathbf{4}$. ${ }^{\mathrm{a}} \mathbf{n}$ was recovered in $50 \%$ yield.

$\mathrm{ZnI}_{2}$ as the model substrate and hydrogen atom donor, respectively.

To investigate the chelation mode of $\mathrm{ZnI}_{2}$ with Int-III, a variety of possible complexes with different chelation modes were extensively screened. The results revealed that the most favored chelation mode contains a $\mathrm{ZnI}_{2}$-chelated six-membered ring, where $\mathrm{ZnI}_{2}$ coordinates with both the carbonyl oxygen atom and the $\mathrm{B}-\mathrm{H}$ bond ${ }^{58,59}$ (Int-III-B-1 in Fig. 7a). ${ }^{1} \mathrm{H}$ NMR spectroscopy of 2a titrated with $\mathrm{ZnI}_{2}$ showed a downfield shift of B-H signals (Supplementary Fig. 2), providing an evidence for the interaction of $\mathrm{ZnI}_{2}$ with the $\mathrm{B}-\mathrm{H}$ bond. Some other chelation models were also considered and computed, such as the coordination of $\mathrm{ZnI}_{2}$ with only methoxycarbonyl oxygen atom (Int-III-B-2), or with the triazole nitrogen atom (Int-III-B-3), or with both the carbonyl group and the indoline nitrogen atom (IntIII-B-4). However, all of them are energetically disfavored in comparison with Int-III-B-1. The HAT transition states of the aforementioned four possible intermediates were located, and a preference of Int-III-B-1 was observed (Supplementary Fig. 5). Moreover, our experimental findings showed no evident difference of $\mathrm{dr}$ value between 2a (7:93, Table 2, entry 9) and 2d (6:94, Table 2, entry 1), hinting that the chelation between $\mathrm{ZnI}_{2}$ and the triazole moiety is most likely not the leading factor of determining HAT diastereoselectivity. However, it is worth mentioning that the use of $\mathbf{2 d}$ (Table 2, entry 1) gives a much better product yield than that of $\mathbf{2 a}$ (Table 2, entry 11), meaning that the dynamic complexation between $\mathbf{2 d}$ and $\mathrm{ZnI}_{2}$ was probably helpful to accelerate the cascade process, while the detailed mechanism is still unclear and further mechanistic studies are currently under progress in our laboratory. In addition, the optimization studies (Table 2) showed that other Lewis acids, such as $\mathrm{MgCl}_{2}, \mathrm{MgI}_{2}, \mathrm{CuI}, \mathrm{ZnCl}_{2}, \mathrm{Me}_{3} \mathrm{Al}$, and $\mathrm{MgBr}_{2} \cdot \mathrm{OEt}_{2}$, are ineffective in controlling the stereoselectivity, suggesting that both the zinc center and the iodide anions are 


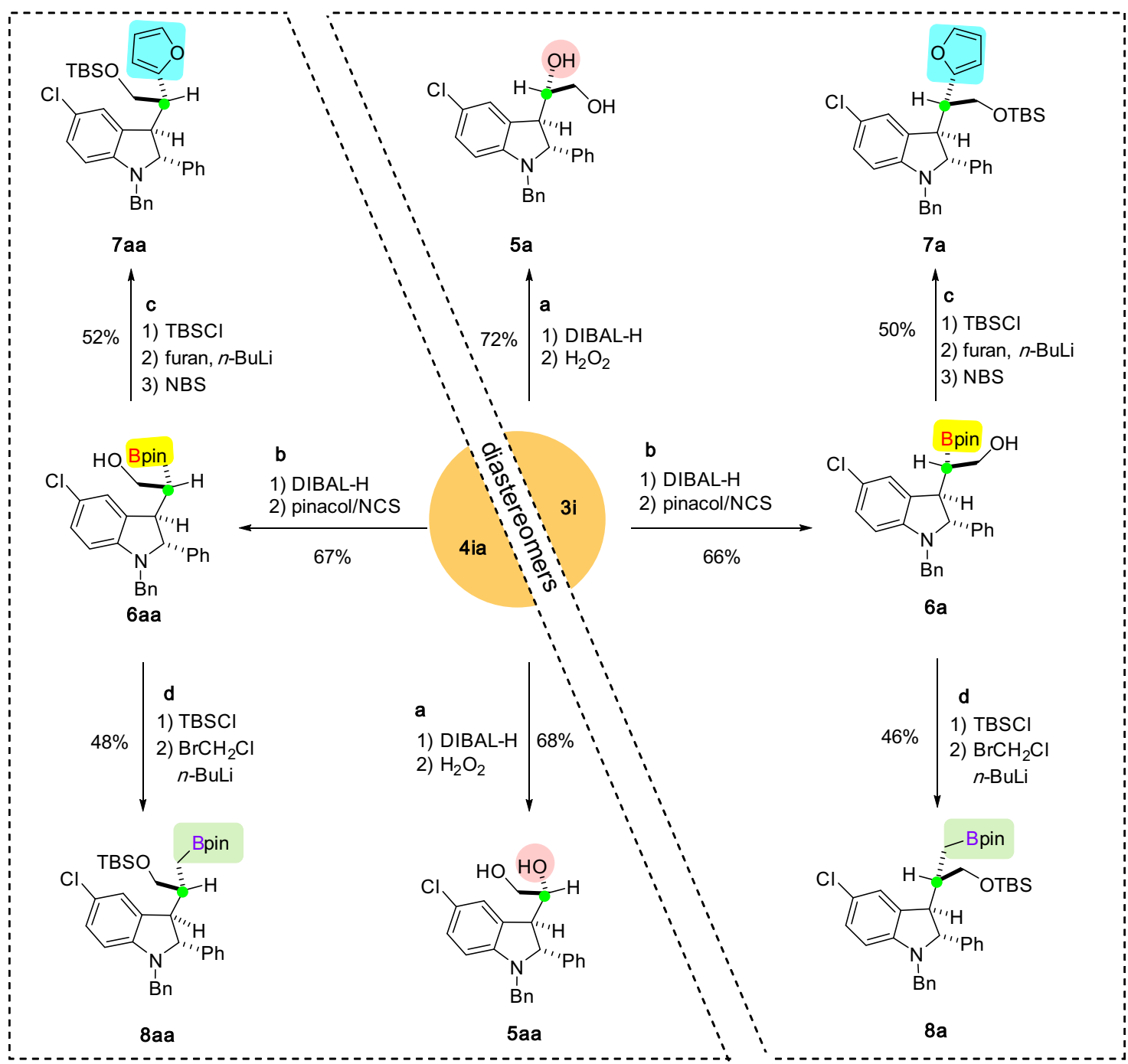

Fig. 4 Stereospecific transformations for the synthesis of diastereomeric functional molecules. a Synthesis of hydroxylated products $\mathbf{5 a}$ and $\mathbf{5 a a}$. $\mathbf{b}$ Synthesis of pinacol boronic esters $\mathbf{6} \mathbf{a}$ and $\mathbf{6} \mathbf{a a}$. $\mathbf{c}$ Synthesis of furan-substituted products $\mathbf{7 a}$ and $\mathbf{7 a a}$. $\mathbf{d}$ Synthesis of homologated pinacol boronic esters $\mathbf{8} \mathbf{a}$ and 8aa.

crucial to form the favored HAT transition state that can lead to high levels of diatereoselectivity. The switch of the anion to chloride might result in a more flexible complexation and the sixmembered ring chelation mode was likely not formed, and then inducing no effect of stereocontrol (Table 2, entry 8).

As depicted in Fig. 7b, calculations suggest HAT from $\mathbf{2 d}-\mathrm{ZnI}_{2}$ complex to Int-III-B-1 prefers to occur via TS-B-major-1 over TS-B-minor-1 $\left(\Delta \Delta G^{\ddagger}=3.3 \mathrm{kcal} / \mathrm{mol}\right)$, which correlates well with the selectivity trend observed in the experiment. There are complexes formed between Int-III-B-1 and $\mathrm{ZnI}_{2}$-ligated 2d, we did not locate them because analyzing the transition states of TSB-major-1 and TS-B-minor-1 can give us answers to stereochemistry. These two transition states TS-B-major-1 and TS-Bminor-1 were then subjected to DIA. Based on the DIA results, the preference of TS-B-major-1 could be mainly ascribed to the lower distortion energy of Int-III-B-1 $(8.5 \mathrm{kcal} / \mathrm{mol})$ than that in TS-B-minor-1 $(11.7 \mathrm{kcal} / \mathrm{mol})$. Careful inspection of the transition state structures reveals the specific Zn-chelated sixmembered ring plays a critical role in controlling the diastereoselectivity. In TS-B-minor-1, the six-membered ring has to undergo substantial torsion to orient towards $N$-Bn group, thus the steric congestion between these two components is likely the cause of high distortion energy in Int-III-B-1. This hypothesis is in line with the poor diastereoselectivities observed in the reactions of $\mathbf{1 b}, \mathbf{1}, \mathbf{1}, \mathbf{n}$, where $N$-alkyl and $N$-phenyl groups are employed instead of $N$-benzyl group (Fig. 3). Subsequent DFT calculation and DIA on HAT step of $\mathbf{1 b}$ provided computational support for this hypothesis, finding that replacing $N$-Bn with $N$ $\mathrm{Me}$ results in a dramatic decrease of the distortion energy (Supplementary Fig. 6). Computational studies of the HAT from free $\mathrm{NHC}-\mathrm{BH}_{3}$ 2d to Int-III-B were also conducted, and the results show that the use of free $\mathbf{2 d}$ or $\mathbf{2} \mathbf{d}-\mathrm{ZnI}_{2}$ complex as the hydrogen atom donor has a negligible effect on the diastereoselectivity (Supplementary Fig. 7).

In summary, we have developed an NHC-boryl radical triggered radical cascade to assemble heterocyclic molecules anchoring a boron-substituted acyclic stereocenter. The stereochemistry of HAT to acyclic radical intermediates is determined by $\mathrm{NHC}-\mathrm{BH}_{3} /$ thiol catalyst and $\mathrm{NHC}-\mathrm{BH}_{3} / \mathrm{ZnI}_{2}$-chelation systems. Experimental and computational studies suggest that these two modes operate in complementary ways. A cooperative control by the NHC unit and a thiol catalyst favor the formation of one diastereomer, while a $\mathrm{B}-\mathrm{H}$ bond-involved six-membered ring chelation with $\mathrm{ZnI}_{2}$ is beneficial for the formation of the other one. Such a stereoselectivity switch enables the divergent synthesis of diastereomeric borylated products. We expect that 


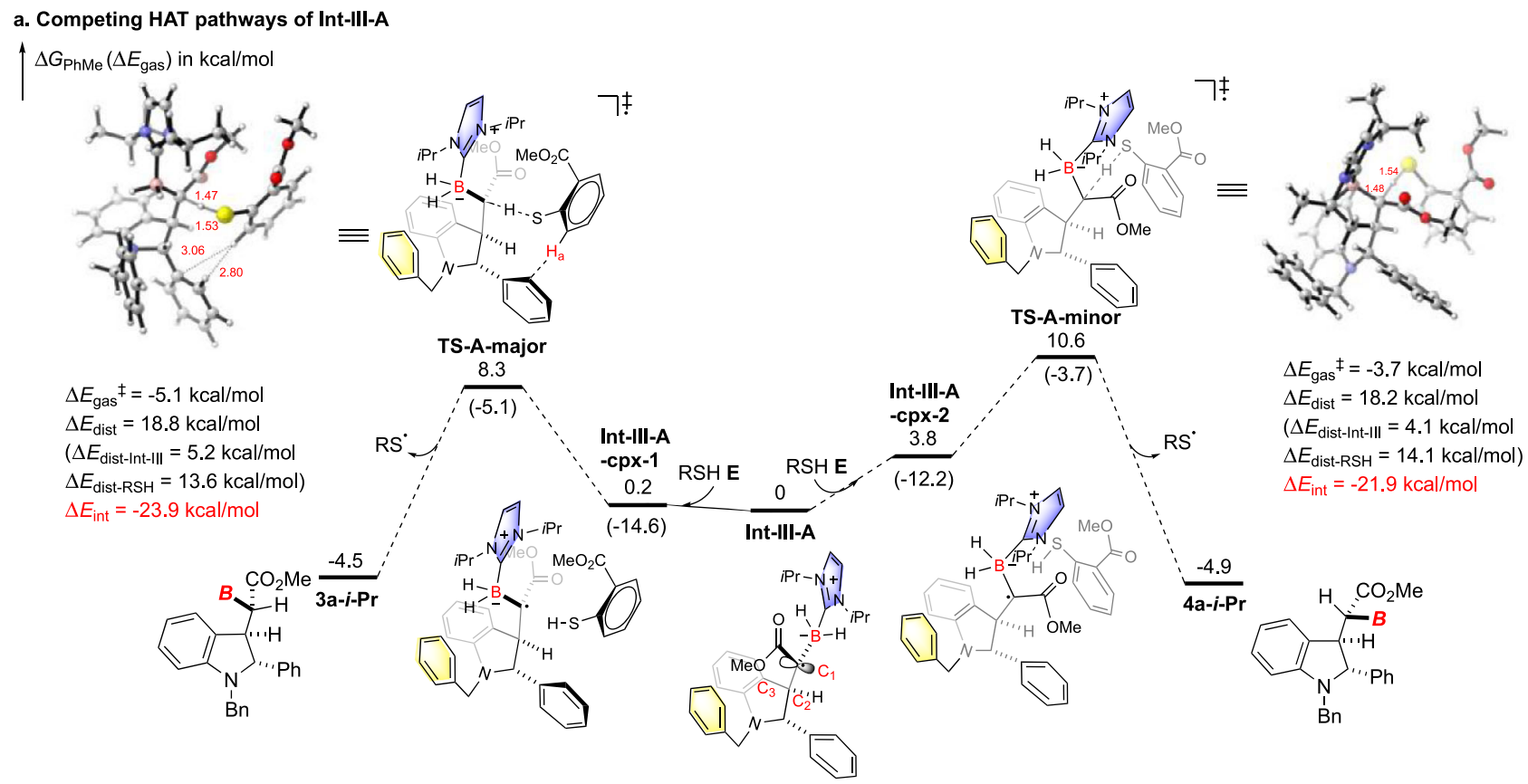

b. $\mathrm{NCl}$ plots of TS-A-major and TS-A-minor

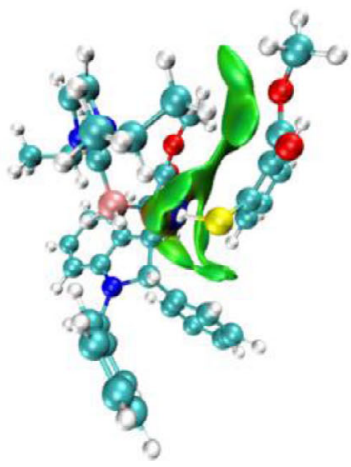

TS-A-major

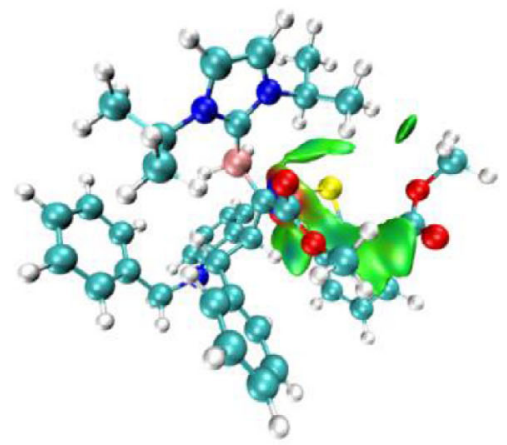

TS-A-minor

Fig. 5 Analysis of the diastereoselectivity in the $\mathbf{N H C}-\mathbf{B H}_{\mathbf{3}} \mathbf{2}$ b/thiol E-controlled $\mathbf{H A T}$ process. a Competing HAT pathways of Int-III-A. b NCI plots of TS-A-major and TS-A-minor.

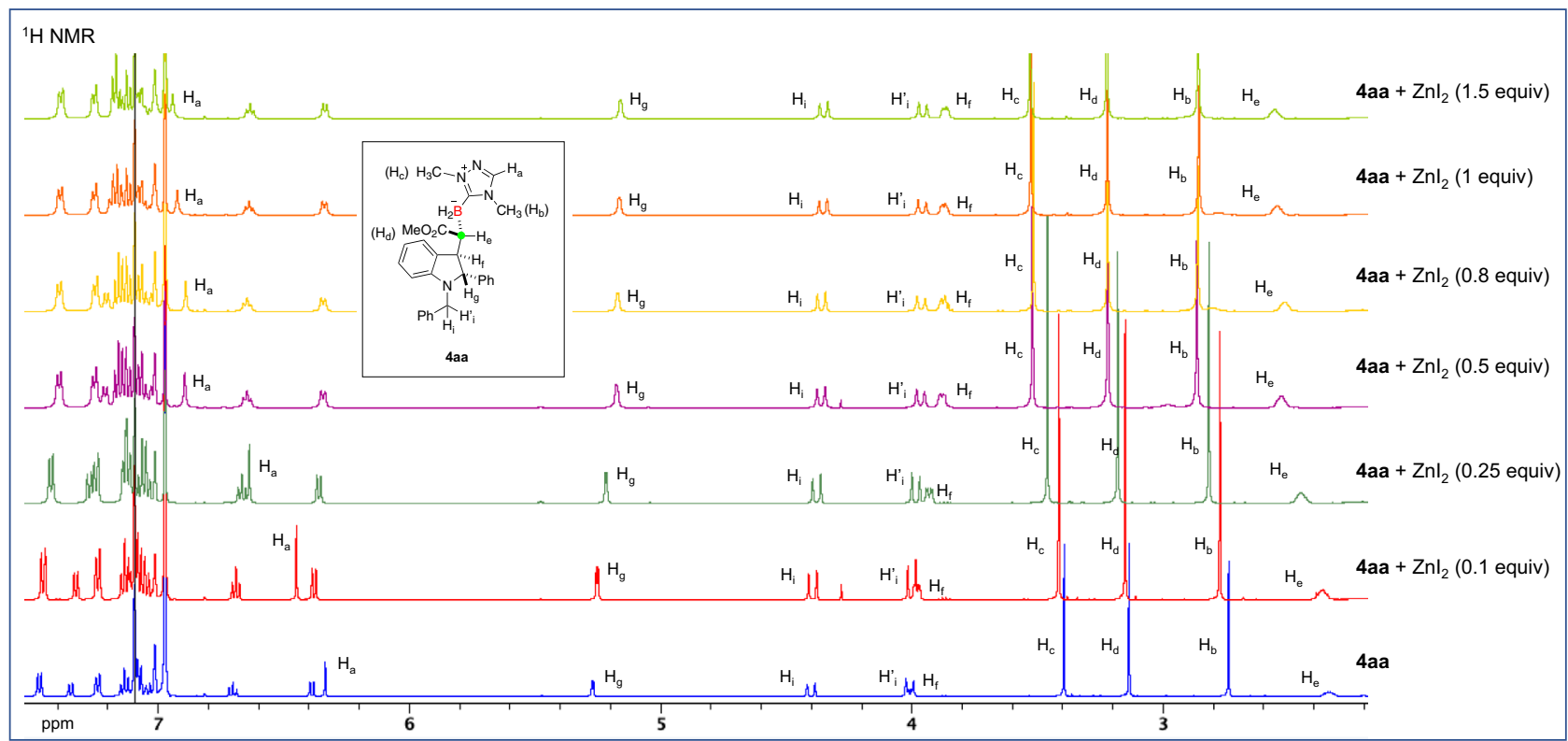

Fig. 6 Stacked ${ }^{\mathbf{1}_{\mathbf{H}}}$ NMR spectra of $\mathbf{4 a a}$ with an increasing amount of $\mathbf{Z n l}_{\mathbf{2}}$. NMR spectra were recorded on a Bruker Avance 500 spectrometers $(500 \mathrm{MHz})$ in toluene- $d_{8}$. 


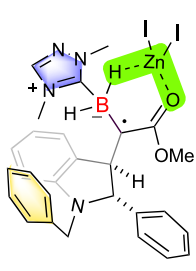

Int-III-B-1

$(\Delta G=0)$

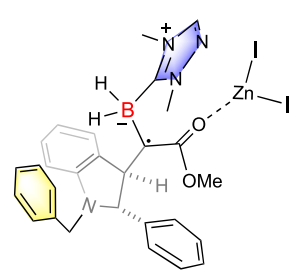

Int-III-B-2

$(\Delta G=3.0)$

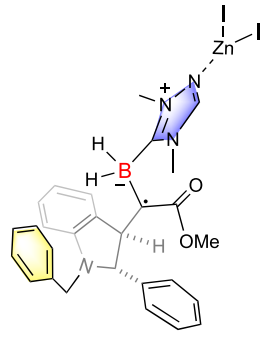

Int-III-B-3

$(\Delta G=6.6)$

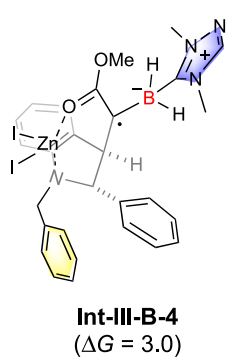

Int-III-B-4
$(\Delta G=3.0)$

b

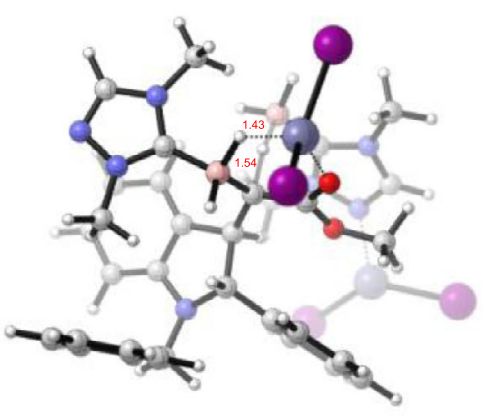

||

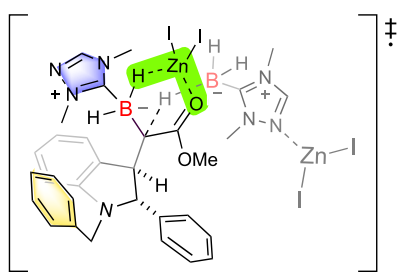

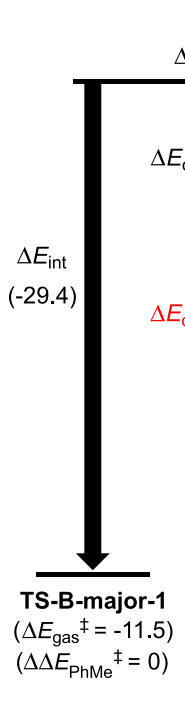

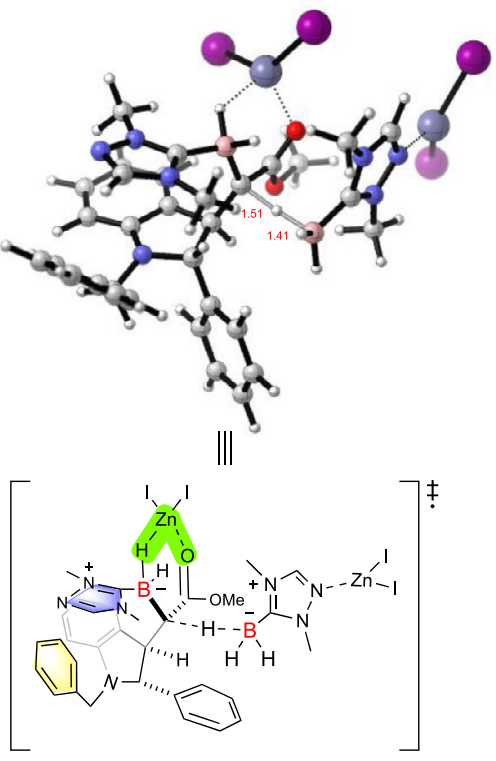

Fig. 7 Computational studies of $\mathbf{Z n I}_{\mathbf{2}}$-mediated reactions. a Int-III-B with different chelation modes. $\mathbf{b}$ Analysis of the diastereoselectivity in the NHC$\mathrm{BH}_{3} / \mathrm{ZnI}_{2}$-controlled HAT processes.

the present stereoselectivity-switchable HAT strategy and the resulting diastereomeric borylated products would be important for synthetic and medicinal applications.

\section{Methods}

General procedure for $\mathrm{NHC}^{-\mathrm{BH}_{3}}$ /thiol catalyst-controlled stereoselective synthesis of borylated molecules. To a $25 \mathrm{~mL}$ flame-dried Schlenk flask under nitrogen, substrate $\mathbf{1}(0.400 \mathrm{mmol}), \mathbf{2 b}(0.480 \mathrm{mmol}), \mathrm{ABVN}(0.080 \mathrm{mmol})$, and phenyl 2-mercaptobenzoate $(0.080 \mathrm{mmol})$ in toluene $(4 \mathrm{~mL})$ was stirred at $60^{\circ} \mathrm{C}$ for 3-12 $\mathrm{h}$ under nitrogen atmosphere. After evaporation of the solvent, the resulting crude residue was purified by flash column chromatography (silica gel; petroleum ether/ethyl acetate) to give the corresponding products.

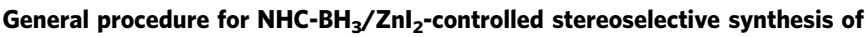
borylated molecules. To a $25 \mathrm{~mL}$ flame-dried Schlenk flask under nitrogen, substrate $1(0.400 \mathrm{mmol}), 2 \mathrm{~d}(0.480 \mathrm{mmol}), \mathrm{ABVN}(0.200 \mathrm{mmol})$, and $\mathrm{ZnI}_{2}$ $(0.400 \mathrm{mmol})$ in toluene $(2 \mathrm{~mL})$ was stirred at $60^{\circ} \mathrm{C}$ for $12-24 \mathrm{~h}$ under nitrogen atmosphere. The reaction mixture was quenched with $\mathrm{NH}_{4} \mathrm{Cl}-\mathrm{NH}_{3} / \mathrm{H}_{2} \mathrm{O}$ buffer solutions. The aqueous layer was extracted three times with dichloromethane. The combined extracts were dried over $\mathrm{Na}_{2} \mathrm{SO}_{4}$ and concentrated in vacuo. The resulting crude residue was purified by flash column chromatography (silica gel; petroleum ether/ethyl acetate) to give the corresponding products.

Computational methods. Calculations were carried out with Gaussian 16 software packages. The (U)B3LYP functional was used with an empirical dispersion correction (Grimme-D3(0)) for all the calculations. The geometries of all the stationary points were optimized in the gas phase with the SDD basis set (Stuttgart/
Dresden ECP) for $\mathrm{Zn}$ and I, and the 6-31G(d,p) basis set for the other atoms. In the computational studies of $\mathrm{HAT}$ controlled by $\mathrm{NHC}-\mathrm{BH}_{3} /$ thiol catalyst, the internal six d-type orbitals were used for all elements in geometry optimization part. In the part of diastereoselective HAT controlled by $\mathrm{NHC}-\mathrm{BH}_{3} /$ Lewis acid, the keyword " $5 \mathrm{D}$ " was used to specify that five d-type orbitals were used for all elements in the calculations. Vibrational frequency analysis was calculated at the same level of theory to validate each structure as either a minimum or a transition state. For each transition state, the intrinsic reaction coordinate (IRC) analysis was conducted to ensure that it connects the right reactant and product. To obtain more accurate energies, single-point energies were calculated with a mixed basis set of SDD for $\mathrm{Zn}$ and I, $6-311+G(d, p)$ for all the other atoms. The distortion/interaction analysis was performed with gas-phase single point energies, while all the other energies reported in the main manuscript were obtained from solution-phase single point energies with SMD solvation model (solvent $=$ toluene). Noncovalent interaction (NCI) analysis was performed with Multiwfn by using the IGM method. The results were visualized by VMD software. 3D structures were generated by CYLview. See Supplementary refs. 22-37.

\section{Data availability}

The X-ray crystallographic coordinates for 3a-Me (CCDC 1994057), 3a-i-Pr (CCDC 1994058), 4a-Me (CCDC 1994059), 3s (CCDC 1994060), and 4aa (CCDC 1994061) have been deposited at the Cambridge Crystallographic Data Centre (CCDC). These data can be obtained free of charge from the CCDC via www.ccdc.cam.ac.uk/data_request/cif. The experimental procedures, kinetic studies, computational results, and characterization of all new compounds are provided in the Supplementary Information.

Received: 26 July 2021; Accepted: 4 January 2022; Published online: 20 January 2022 


\section{References}

1. Carreira, E. M. \& Kvaerno, L. Classics in Stereoselective Synthesis (Wiley-VCH, 2009).

2. Poppe, L. \& Nógrádi, M. Stereochemistry and Stereoselective Synthesis: An Introduction (Wiley-VCH, 2016).

3. Xuan, J. \& Studer, A. Radical cascade cyclization of $1, n$-enynes and diynes for the synthesis of carbocycles and heterocycles. Chem. Soc. Rev. 46, 4329-4346 (2017).

4. Wille, U. Radical cascades initiated by intermolecular radical addition to alkynes and related triple bond systems. Chem. Rev. 113, 813-853 (2013).

5. Baralle, A. et al. in Encyclopedia of Radicals in Chemistry, Biology and Materials (eds Chatgilialoglu, C. \& Studer, A.) (John Wiley \& Sons, Ltd 2012).

6. Curran, D. P., Porter, N. A. \& Giese, B. Stereochemistry of Radical Reactions: Concepts, Guidelines, and Synthetic Applications (1996).

7. Porter, N. A., Giese, B. \& Curran, D. P. Acyclic stereochemical control in freeradical reactions. Acc. Chem. Res. 24, 296-304 (1991).

8. Beletskaya, I. P., Nájera, C. \& Yus, M. Stereodivergent catalysis. Chem. Rev. 118, 5080-5200 (2018)

9. Gansaeuer, A. et al. in Radicals in Synthesis III (eds Heinrich, M. R. \& Gansauer, A.) 93-120 (2012)

10. Barth, F., Achrainer, F., Pütz, A. M. \& Zipse, H. Aminopyridine-borane complexes as hydrogen atom donor reagents: reaction mechanism and substrate selectivity. Chem. Eur. J. 23, 13455-13464 (2017).

11. Kawamoto, T. \& Ryu, I. Radical reactions of borohydrides. Org. Biomol. Chem. 12, 9733-9742 (2014).

12. Godin, F. et al. Diastereoselective hydrogen-transfer reactions: an experimental and DFT study. Chem. Eur. J. 19, 9308-9318 (2013).

13. Gansäuer, A., Klatte, M., Brändle, G. M. \& Friedrich, J. Catalytic hydrogen atom transfer (HAT) for sustainable and diastereoselective radical reduction. Angew. Chem. Int. Ed. 51, 8891-8894 (2012).

14. Guindon, Y. et al. The exocyclic effect: protecting group strategy to enhance stereoselectivity in hydrogen transfer reactions of acyclic free radicals. J. Org. Chem. 62, 9276-9283 (1997).

15. Durkin, K. et al. Stereoselective hydrogen-transfer reactions involving acyclic radicals. A study of radical conformations using semiempirical calculations. J. Am. Chem. Soc. 114, 4912-4914 (1992).

16. Renaud, P. \& Gerster, M. Use of Lewis acids in free radical reactions. Angew. Chem. Int. Ed. 37, 2562-2579 (1998).

17. Miyabe, H. \& Takemoto, Y. Enantioselective radical cyclizations: a new approach to stereocontrol of cascade reactions. Chem. Eur. J. 13, 7280-7286 (2007).

18. Curran, D. P., Shen, W., Zhang, J. \& Heffner, T. A. Asymmetric radical addition, cyclization, and annulation reactions with Oppolzer's camphor sultam. J. Am. Chem. Soc. 112, 6738-6740 (1990).

19. Sibi, M. P. \& Rheault, T. R. Higher selectivity at higher temperatures! Effect of precursor stereochemistry on diastereoselectivity in radical allylations. Insight into the role of the Lewis acid. J. Am. Chem. Soc. 122 8873-8879 (2000)

20. Godin, F. et al. Stereocontrolled synthesis of propionate motifs froml-lactic andl-alanine aldehydes. A DFT study of the hydrogen transfer under endocyclic control. Org. Chem. Front. 1, 974-982 (2014).

21. Ueng, S.-H. et al. Complexes of borane and $N$-heterocyclic carbenes: a new class of radical hydrogen atom donor. J. Am. Chem. Soc. 130, 10082-10083 (2008)

22. Watanabe, T., Hirose, D., Curran, D. P. \& Taniguchi, T. Borylative radical cyclizations of benzo[3,4]cyclodec-3-ene-1,5-diynes and $N$-heterocyclic carbene-boranes. Chem. Eur. J. 23, 5404-5409 (2017).

23. Shimoi, M., Watanabe, T., Maeda, K., Curran, D. P. \& Taniguchi, T. Radical trans-hydroboration of alkynes with $\mathrm{N}$-heterocyclic carbene boranes. Angew. Chem. Int. Ed. 57, 9485-9490 (2018).

24. Dai, W., Geib, S. J. \& Curran, D. P. Facile synthesis of $a-N$-heterocyclic carbene-boryl ketones from $N$-heterocyclic carbene-boranes and alkenyl triflates. J. Am. Chem. Soc. 141, 12355-12361 (2019).

25. Shimoi, M. et al. The Thermal rearrangement of an NHC-ligated 3-benzoborepin to an NHC-boranorcaradiene. Angew. Chem. Int. Ed. 59, 903-909 (2020).

26. Jin, J., Xia, H., Zhang, F. \& Wang, Y. Lewis-base boryl radicals enabled borylation, radical catalysis and reduction reactions. Chin. J. Org. Chem. 40, 2185-2194 (2020).

27. Ren, S.-C. et al. Radical borylation/cyclization cascade of 1,6-enynes for the synthesis of boron-handled hetero- and carbocycles. J. Am. Chem. Soc. 139, 6050-6053 (2017).

28. Ren, S.-C. et al. Regioselective radical $\alpha$-borylation of $\alpha, \beta$-unsaturated carbonyl compounds for direct synthesis of a-borylcarbonyl molecules. Nat. Commun. 10, 1934 (2019).

29. Qi, J. et al. New radical borylation pathways for organoboron synthesis enabled by photoredox catalysis. Angew. Chem. Int. Ed. 59, 12876-12884 (2020).

30. Zhou, N., Yuan, X.-A., Zhao, Y., Xie, J. \& Zhu, C. Synergistic photoredox catalysis and organocatalysis for inverse hydroboration of imines. Angew. Chem. Int. Ed. 57, 3990-3994 (2018).
31. Zhu, C. et al. Photoredox-controlled $\beta$-regioselective radical hydroboration of activated alkenes with NHC-boranes. Angew. Chem. Int. Ed. 59, 12817-12821 (2020).

32. Xu, W., Jiang, H., Leng, J., Ong, H. W. \& Wu, J. Visible-light-induced selective defluoroborylation of polyfluoroarenes, gem-difluoroalkenes, and trifluoromethylalkenes. Angew. Chem. Int. Ed. 59, 4009-4016 (2020).

33. Kawamoto, T., Morioka, T., Noguchi, K., Curran, D. P. \& Kamimura, A. Inverse hydroboration of imines with NHC-boranes is promoted by diphenyl disulfide and visible light. Org. Lett. 23, 1825-1828 (2021).

34. Roberts, B. P. Polarity-reversal catalysis of hydrogen-atom abstraction reactions: concepts and applications in organic chemistry. Chem. Soc. Rev. 28, 25-35 (1999).

35. Pan, X., Lacôte, E., Lalevée, J. \& Curran, D. P. Polarity reversal catalysis in radical reductions of halides by $\mathrm{N}$-heterocyclic carbene boranes. J. Am. Chem. Soc. 134, 5669-5674 (2012).

36. Curran, D. P., Kim, D., Liu, H. T. \& Shen, W. Translocation of radical sites by intramolecular 1,5-hydrogen atom transfer. J. Am. Chem. Soc. 110, 5900-5902 (1988).

37. Curran, D. P. \& Shen, W. Radical translocation reactions of vinyl radicals: substituent effects on 1,5-hydrogen-transfer reactions. J. Am. Chem. Soc. 115, 6051-6059 (1993).

38. Robertson, J., Pillai, J. \& Lush, R. K. Radical translocation reactions in synthesis. Chem. Soc. Rev. 30, 94-103 (2001).

39. Denes, F., Beaufils, F. \& Renaud, P. Preparation of five-membered rings via the translocation-cyclization of vinyl radicals. Synlett 16, 2389-2399 (2008).

40. Welsch, M. E., Snyder, S. A. \& Stockwell, B. R. Privileged scaffolds for library design and drug discovery. Curr. Opin. Chem. Biol. 14, 347-361 (2010).

41. Norwood, V. M. IV \& Huigens, R. W. III Harnessing the chemistry of the indole heterocycle to drive discoveries in biology and medicine. ChemBioChem 20, 2273-2297 (2019).

42. Namirembe, S. \& Morken, J. P. Reactions of organoboron compounds enabled by catalyst-promoted metalate shifts. Chem. Soc. Rev. 48, 3464-3474 (2019)

43. Leonori, D. \& Aggarwal, V. K. Stereospecific couplings of secondary and tertiary boronic esters. Angew. Chem. Int. Ed. 54, 1082-1096 (2015).

44. Fyfe, J. W. B. \& Watson, A. J. B. Recent developments in organoboron chemistry: old dogs, new tricks. Chem 3, 31-55 (2017).

45. Takahashi, K., Geib, S. J., Maeda, K., Curran, D. P. \& Taniguchi, T. Radical trans-hydroboration of substituted 1,3-diynes with an $\mathrm{N}$-heterocyclic carbene borane. Org. Lett. 23, 1071-1075 (2021).

46. Watanabe, T., Geib, S. J., Curran, D. P. \& Taniguchi, T. N-Heterocyclic carbene boranes are hydrogen donors in Masamune-Bergman reactions of benzo[3,4]cyclodec-3-ene-1,5-diynes. J. Org. Chem. 82, 13034-13042 (2017)

47. Solovyev, A. et al. Estimated rate constants for hydrogen abstraction from $N$ heterocyclic carbene-borane complexes by an alkyl radical. Org. Lett. 12, 2998-3001 (2010).

48. Tambutet, G. \& Guindon, Y. Diastereoselective radical hydrogen transfer reactions using $N$-heterocyclic carbene boranes. J. Org. Chem. 81, 11427-11431 (2016).

49. Bonet, A., Odachowski, M., Leonori, D., Essafi, S. \& Aggarwal, V. K. Enantiospecific $\mathrm{sp}^{2}-\mathrm{sp}^{3}$ coupling of secondary and tertiary boronic esters. Nat. Chem. 6, 584 (2014).

50. Seeman, J. I. Effect of conformational change on reactivity in organic chemistry. Evaluations, applications, and extensions of Curtin-Hammett Winstein-Holness kinetics. Chem. Rev. 83, 83-134 (1983).

51. Bickelhaupt, F. M. \& Houk, K. N. Analyzing reaction rates with the distortion/ interaction-activation strain model. Angew. Chem. Int. Ed. 56, 10070-10086 (2017).

52. Fernández, I. \& Bickelhaupt, F. M. The activation strain model and molecular orbital theory: understanding and designing chemical reactions. Chem. Soc. Rev. 43, 4953-4967 (2014).

53. van Zeist, W.-J. \& Bickelhaupt, F. M. The activation strain model of chemical reactivity. Org. Biomol. Chem. 8, 3118-3127 (2010).

54. Johnson, E. R. et al. Revealing noncovalent interactions. J. Am. Chem. Soc 132, 6498-6506 (2010).

55. Lefebvre, C. et al. Accurately extracting the signature of intermolecular interactions present in the NCI plot of the reduced density gradient versus electron density. Phys. Chem. Chem. Phys. 19, 17928-17936 (2017).

56. Williams, J. H. The molecular electric quadrupole moment and solid-state architecture. Acc. Chem. Res. 26, 593-598 (1993).

57. Umezawa, Y., Tsuboyama, S., Takahashi, H., Uzawa, J. \& Nishio, M. CH/ $\pi$ interaction in the conformation of organic compounds. A database study. Tetrahedron 55, 10047-10056 (1999).

58. Bissinger, P., Braunschweig, H., Kupfer, T. \& Radacki, K. Monoborane NHC adducts in the coordination sphere of transition metals. Organometallics 29, 3987-3990 (2010)

59. Saha, K., Roy, D. K., Dewhurst, R. D., Ghosh, S. \& Braunschweig, H. Recent advances in the synthesis and reactivity of transition metal $\sigma$-borane/borate complexes. Acc. Chem. Res. 54, 1260-1273 (2021). 


\section{Acknowledgements}

This work is supported by the National Natural Science Foundation of China (21971226 and 21933003) and the Fundamental Research Funds for the Central Universities (WK2060000017). The numerical calculations in this study have been done on the supercomputing system in the Super-computing Center of the University of Science and Technology of China. We thank Mr. Yi Zhou (Peking University) for valuable discussions on computational studies and careful proofreading of the manuscript.

\section{Author contributions}

T.Y. and F.-L.Z. contributed equally to this work. T.Y., H.-M.X., and X.Z. performed all the experiments. F.-L.Z. conducted the DFT calculations and provided mechanism analysis. Y.Z.X. directed the DFT calculations and mechanism analysis. Y.-F.W. supervised the project. F.-L.Z., Y.-Z.X., and Y.-F.W. wrote the manuscript with the help of all co-authors.

\section{Competing interests}

The authors declare no competing interests.

\section{Additional information}

Supplementary information The online version contains supplementary material available at https://doi.org/10.1038/s41467-022-28071-8.

Correspondence and requests for materials should be addressed to Zhi-Xiang Yu or Yi-Feng Wang.
Peer review information Nature Communications thanks Tsuyoshi Taniguchi and the other anonymous reviewer(s) for their contribution to the peer review of this work.

Reprints and permission information is available at http://www.nature.com/reprints

Publisher's note Springer Nature remains neutral with regard to jurisdictional claims in published maps and institutional affiliations.

(c) (i) Open Access This article is licensed under a Creative Commons Attribution 4.0 International License, which permits use, sharing, adaptation, distribution and reproduction in any medium or format, as long as you give appropriate credit to the original author(s) and the source, provide a link to the Creative Commons license, and indicate if changes were made. The images or other third party material in this article are included in the article's Creative Commons license, unless indicated otherwise in a credit line to the material. If material is not included in the article's Creative Commons license and your intended use is not permitted by statutory regulation or exceeds the permitted use, you will need to obtain permission directly from the copyright holder. To view a copy of this license, visit http://creativecommons.org/ licenses/by/4.0\%.

(C) The Author(s) 2022 